\title{
Hardware Implementation Aspects of New Low Complexity Image Coding Algorithm for Wireless Capsule Endoscopy
}

\author{
Paweł Turcza ${ }^{1,4}$, Tomasz Zieliński ${ }^{2,4}$, and Mariusz Duplaga ${ }^{3,4}$ \\ ${ }^{1}$ Department of Instrumentation and Measurement, ${ }^{2}$ Department of Telecommunications, \\ AGH University of Science and Technology, Kraków, Poland \\ ${ }^{3}$ Collegium Medicum, Jagiellonian University, Kraków, Poland \\ ${ }^{4}$ Center of Innovation, Technology Transfer and University Development, \\ Jagiellonian University, Kraków, Poland \\ \{turcza,tzielin\}@agh.edu.pl, mmduplag@cyf-kr.edu.pl
}

\begin{abstract}
The paper presents hardware implementation aspects of new efficient image compression algorithm designed for wireless capsule endoscopy with Bayer color filter array (CFA). Since power limitation, small size conditions and specific image data format (CFA) exclude application of traditional image compression techniques dedicated ones are necessary. Discussed algorithm is based on integer version of discrete cosine transform (DCT). Therefore it has low complexity and power consumption. It is demonstrated that the performance of proposed algorithm is comparable to the performance of JPEG2000 very complex, sophisticated wavelet based coder. In the paper a VLSI coder architecture is proposed and power requirements are discussed.
\end{abstract}

Keywords: Image coding, integer transformation, DCT, Bayer, JPEG2000.

\section{Introduction}

An endoscopic medical procedure, often accompanied by a biopsy of pathological changes, plays a fundamental role in diagnosis of many gastrointestinal (GI) tract diseases. Until recently it has been the most important method of investigation of upper (gastroscopy) and lower (colonoscopy) parts of the GI tract. However, examination with flexible endoscope is also very unpleasant experience for a patient.

Due to enormous progress in microelectronics a wireless endoscopic capsule has been invented recently that makes possible a non-invasive evaluation of the whole GI tract together will small intestine. First such capsule was built by Given Imaging Ltd. [1] in the end of 20-th century. It is equipped with a CMOS sensor, lighting, data processing module and transmission unit (Fig. 1). After swallowing by a patient, the capsule is passing passively through the GI tract due to peristaltic intestine movements and making photos (images) that are wirelessly transmitted to the recorder carried by a patient. Unfortunately, this technology is not ideal yet. Due to lack of autonomous locomotion and navigation system, detail investigation of the highvolume stomach is impossible, whereas detail investigation of large intestine is 
possible only after its inflation since normally it is shrinked. Quality of the recorded video ( $256 \times 256$ pixels, 8 bits per color, only 2 frames per second) is very low also.

It has been already reported in literature [2], [3] that the endoscopic capsule can approximately reach only one megabit per second transmission bitrate due to limitation of power consumption and severe attenuation of radio waves in a human body. Because the CMOS sensor having VGA resolution $(640 \times 480$ pixels) delivers $2.45 \times 10^{6}$ bits per image, it is obvious that without data compression transmission of one image would last more than 2 seconds spending quite a lot of energy.

Since existing standard image compression algorithms are not appropriate for capsule endoscopy (CE) due to their high computational complexity, simple dedicated algorithms are under development [3], [4]. In this paper memory requirements optimization and hardware implementation aspects of a recently proposed algorithm [5], [6], better than [3], [4], are presented. Influence of codebook reduction in entropy coder on algorithm performance is carefully evaluated and justified. It is shown that the performance of such a modified algorithm is comparable to the performance of JPEG2000 - very complex wavelet based coder. It should be mentioned that problems associated with color filter arrays (CFA) Bayer pattern are efficiently solved by our algorithm, in contrary to the approach presented in [3]. Our method makes it also possible to reconstruct more precisely G1 and G2 CFA image color components. In contrary to [4] our algorithm offers higher compression range, so higher image frame rate is possible.

In the paper a VLSI coder architecture is proposed and power requirements are discussed.

\section{New Image Coding Algorithm}

Simplified block diagram of data processing in wireless endoscopy capsule is presented in Fig. 1. The output image data from a CMOS image sensor after image and channel coding (required for bandwidth reduction and error protection) are transmitted by a wireless transceiver (TX) to the outside of the body where they are received, stored and eventually decompressed for subsequent diagnosis. A control unit in the capsule controls operation of a compression module according to commands received from an external controller.

Proposed image coder performs sequentially four operations: color transformation, image transformation, coefficients quantization and entropy coding.

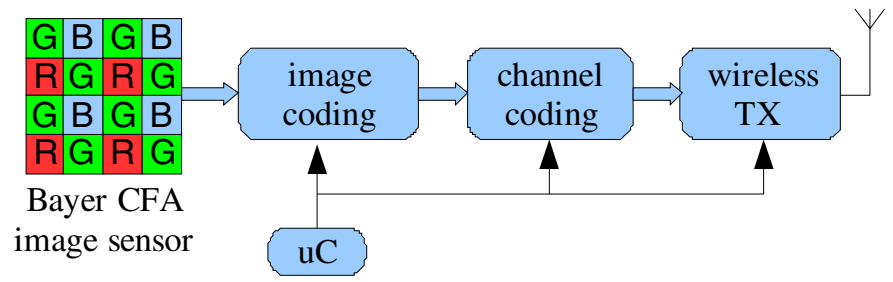

Fig. 1. Block diagram of data processing in wireless endoscopy capsule 


\subsection{Color and Structure Transformation}

In the $\mathrm{CE}$ as well as in inexpensive digital cameras, color filters arrays (CFA) are placed on monochrome CMOS image sensor to produce color images. The most popular CFA pattern has been proposed by Bayer [7]. The Bayer CFA, shown in Fig. 2, uses $2 \times 2$ repeating patterns having two green pixels, one red and one blue. It is clear that application of the CFA results in collection of incomplete image information (only one color component for each pixel) so color interpolation is necessary to reconstruct the full color image from the sensor data. From image compression point of view interpolation step introduces redundancy that is difficult to remove. Therefore, the image compression should precede color data interpolation step for best performance [8].

Additional redundancy reduction can be achieved by a color space transformation. For normal RGB images this is get by the following one:

$$
\left[\begin{array}{l}
Y \\
C_{b} \\
C_{r}
\end{array}\right]=\left[\begin{array}{lll}
0.299 & 0.587 & 0.114 \\
-0.146 & -0.288 & -0.434 \\
0.617 & -0.517 & 0.100
\end{array}\right]\left[\begin{array}{l}
R \\
G \\
B
\end{array}\right]
$$

where $\mathrm{Y}$ is luma component while $\mathrm{Cb}$ and $\mathrm{Cr}$ denote blue and red chroma components, respectively. However, due to $\mathrm{CE}$ power limits and quincunx sampling scheme of green component in Bayer CFA, we propose application of modified RGB to YCgCo space conversion known from Fidelity Range Extensions (FRExt) of H.264 video coding standard [9]:

$$
\left[\begin{array}{l}
Y_{1} \\
Y_{2} \\
C_{g} \\
C_{o}
\end{array}\right]=\left[\begin{array}{llll}
1 / 2 & 0 & 1 / 4 & 1 / 4 \\
0 & 1 / 2 & 1 / 4 & 1 / 4 \\
1 / 4 & 1 / 4 & -1 / 4 & -1 / 4 \\
0 & 0 & -1 / 2 & 1 / 2
\end{array}\right]\left[\begin{array}{l}
G_{1} \\
G_{2} \\
B \\
R
\end{array}\right]
$$

where the $\mathrm{Cg}$ stands for green chroma and the Co stands for orange chroma.

Quincunx array (luma component) has high frequency content that makes compression task difficult. Therefore structure conversion [8] (see Fig. 2) with optional reversible filtering (deinterlacing) (see Fig. 3) is applied in our coder.

\subsection{Image Data Transformation and Entropy Coding}

Block diagram of a general transform based image coder (for only one image component) is presented in Fig. 4. 2D image transformation is the first performed operation. Its goal is to concentrate image energy in the possibly smallest number of transform coefficients that are then scalar quantized and efficiently coded by entropy encoder. As the image transformation usually discrete cosine (DCT) or wavelet (DWT) transforms are chosen. In the proposed algorithm integer approximation of the DCT transformation is used. 


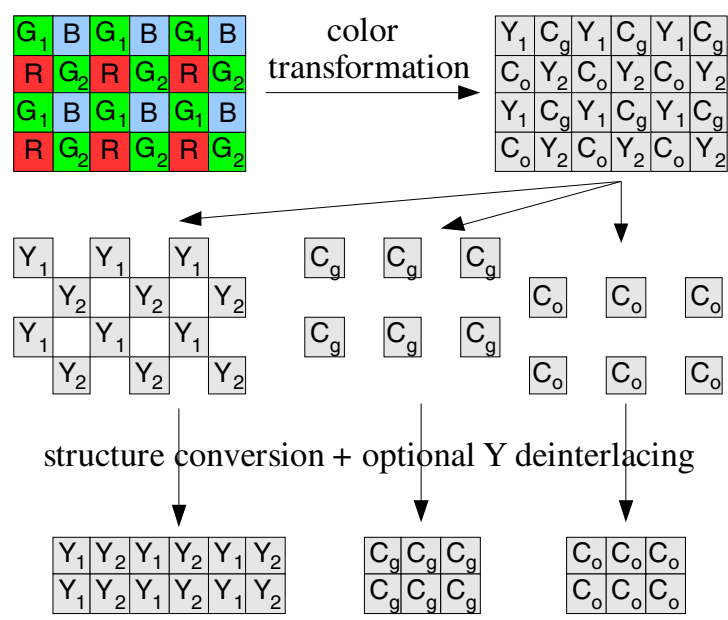

Fig. 2. Bayer CFA and color/structure conversion

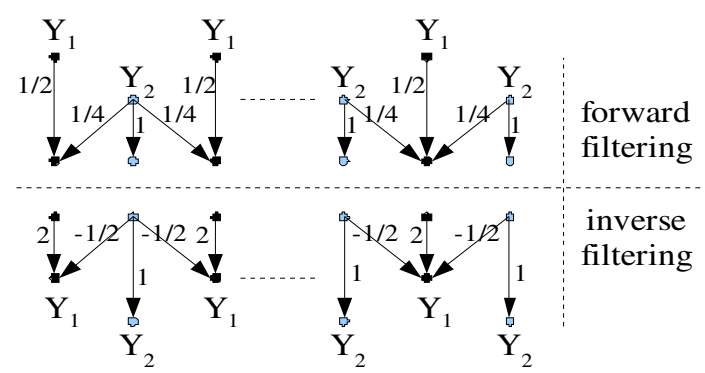

Fig. 3. Reversible image filtering (deinterlacing) [8]

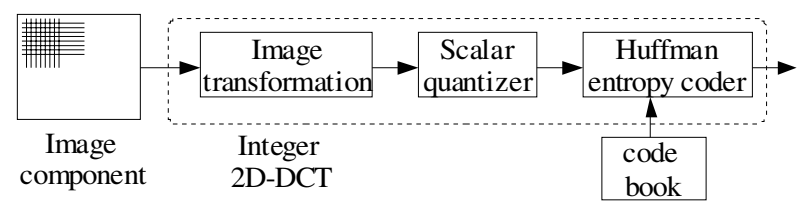

Fig. 4. Block diagram of transform based image coder

The two-dimensional (2D) DCT is a separable transformation and is usually implemented as the 1D row-wise transformation followed by the $1 \mathrm{D}$ column-wise one. In the proposed algorithm the $2 \mathrm{D}$-DCT of each $4 \times 4$ non-overlapped input data block X (separate RGB or YCgCo components) has been computed as

$$
\mathbf{Y}=\mathbf{T}_{f} \mathbf{X} \mathbf{T}_{f}^{T}
$$

where $\mathbf{T}_{f}$ denotes an integer approximation of the 1D-DCT matrix [10]: 


$$
\mathbf{T}_{f}=\left[\begin{array}{llll}
1 & 1 & 1 & 1 \\
2 & 1 & -1 & -2 \\
1 & -1 & -1 & 1 \\
1 & -2 & 2 & -1
\end{array}\right]
$$

and superscript $\mathrm{T}$ is a transposition. Since the transformation (3)(4) represents only an approximation of the original DCT, an additional scaling operation by matrix $\mathrm{S}_{f}$ is required in (3):

$$
\mathbf{Y}=\left(\mathbf{T}_{f} \mathbf{X T}_{f}^{T}\right) \otimes \mathbf{S}_{f}, \quad \mathbf{S}_{f}=\left[\begin{array}{llll}
a^{2} & a b / 2 & a^{2} & a b / 2 \\
a b / 2 & b^{2} / 4 & a b / 2 & b^{2} / 4 \\
a^{2} & a b / 2 & a^{2} & a b / 2 \\
a b / 2 & b^{2} / 4 & a b / 2 & b^{2} / 4
\end{array}\right],
$$

where $a=1 / 2, b=\sqrt{2 / 5}$. and $\otimes$ is the Kronecker product. The element-by-element multiplication $\otimes$ can be incorporated into quantization step.

What is important, apart from good data decorrelation property, the 1D-DCT (4) has also very efficient computational implementation presented in Fig. 5.

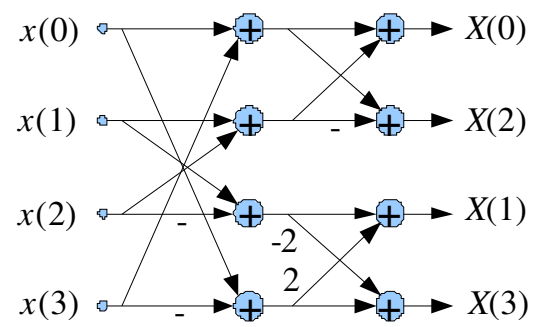

Fig. 5. Low complexity 4-point DCT butterfly algorithm

The inverse DCT of a $4 \times 4$ input data block $\mathbf{Y}$ can be computed using the following formula:

$$
\hat{\mathbf{X}}=\mathbf{T}_{i}^{T}\left(\mathbf{Y} \otimes \mathbf{S}_{i}\right) \mathbf{T}_{i}
$$

where

$$
\mathbf{T}_{i}=\left[\begin{array}{llll}
1 & 1 & 1 & 1 / 2 \\
1 & 1 / 2 & -1 & -1 \\
1 & -1 / 2 & -1 & 1 \\
1 & -1 & 1 & -1 / 2
\end{array}\right], \quad \mathbf{S}_{i}=\left[\begin{array}{llll}
a^{2} & a b & a^{2} & a b \\
a b & b^{2} & a b & b^{2} \\
a^{2} & a b & a^{2} & a b \\
a b & b^{2} & a b & b^{2}
\end{array}\right] .
$$

After the DCT transformation the coefficients of $\mathbf{Y}$ are scalar quantized what results in high compression ratio and loss of information. 
In the discussed algorithm the coefficients of the DCT transform are first quantized and then entropy coded. A technique well known from the JPEG standard [11] was applied to data blocks $4 \times 4$ instead of $8 \times 8$. Since DC coefficients of adjacent $4 \times 4$ blocks are strongly correlated they are coded differentially. The remaining $\mathrm{AC}$ coefficients are entropy coded in a 2-step process. In the first step sequence of quantized coefficients is converted into an intermediate sequence of symbols $(R L, v)$ where $R L$ is the number of consecutive zero-valued AC coefficients in the sequence preceding the nonzero AC coefficient $v$. If all the remaining $\mathrm{AC}$ coefficients in the block are zero they are represented by symbol $(0,0)$. In the second step variable-length Huffman codes (VLC) are assigned to the symbols $z=16 R L+|v|_{2}$ where $|v|_{2}$ denotes length of binary representation (without leading zeros) of $v$ (if $v>0$ ) or $-v$ (if $v<0$ ). Value of $v$ is encoded using variable length integer (VLI) code whose length in bits $|v|_{2}$ was already encoded using VLC. VLI of $v$ equals to binary representation of $v$ if $v>0$ or equals to $-v$ if $v<0$. Separated Huffman tables are designed for DC and AC coefficients.

\subsection{Hardware Implementation}

Finally, designing future hardware implementation of the proposed image encoder has been addressed. A developed VLSI architecture is presented in Fig. 6. As expected, the memory is the most using resources. It is required by the 2D DCT transformation, the color converter and the entropy encoder. Although 1D DCT over rows can be implemented very efficiently (during pixel acquisition) the vertical one requires 4 rows to be stored in memory. Since the color converter operates on two lines, one of them has to be acquired first and stored in memory.

In order to reduce memory required by entropy coder, Huffman codes for symbols $z \geq 64$ are not stored in a code book. Instead they are encoded as a escape sequence follow by fixed length code. Since such symbols occur very infrequently, this technique allows for 4 times reduction of codebook size causing only negligible decrease in compression ratio. The proposed approach significantly reduces Huffman code length as well as width of codebook memory word.

The estimated power budget of the design is given in Tab. 1. For VGA (640x480) image resolution and 10 frames per second (fps) pixel clock is equal to $3 \mathrm{MHz}$. The circuit supply current is $3 \times 0.984=2.95 \mathrm{~mA}$. We can conclude that the expected power requirement are similar to much simple design proposed in [3], but our algorithm offers significantly higher compression ration.

\section{Results}

The proposed algorithm has been implemented as a computer program and its efficiency has been measured. Two exemplary full RGB color images, one from colonoscopy (Fig. 7) and one from gastroscopy (Fig. 8), have been used in initial experimental tests. Since the discussed algorithm operates on CFA Bayer format, the test images have been converted into this format by low-pass filtering (with short 3-tap 


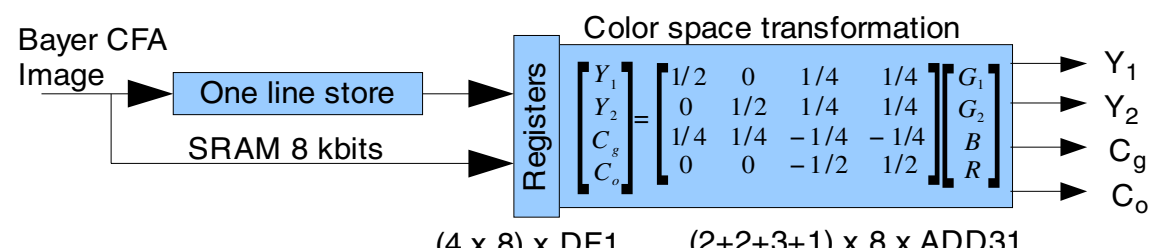

$(4 \times 8) \times$ DF1 $\quad(2+2+3+1) \times 8 \times$ ADD31
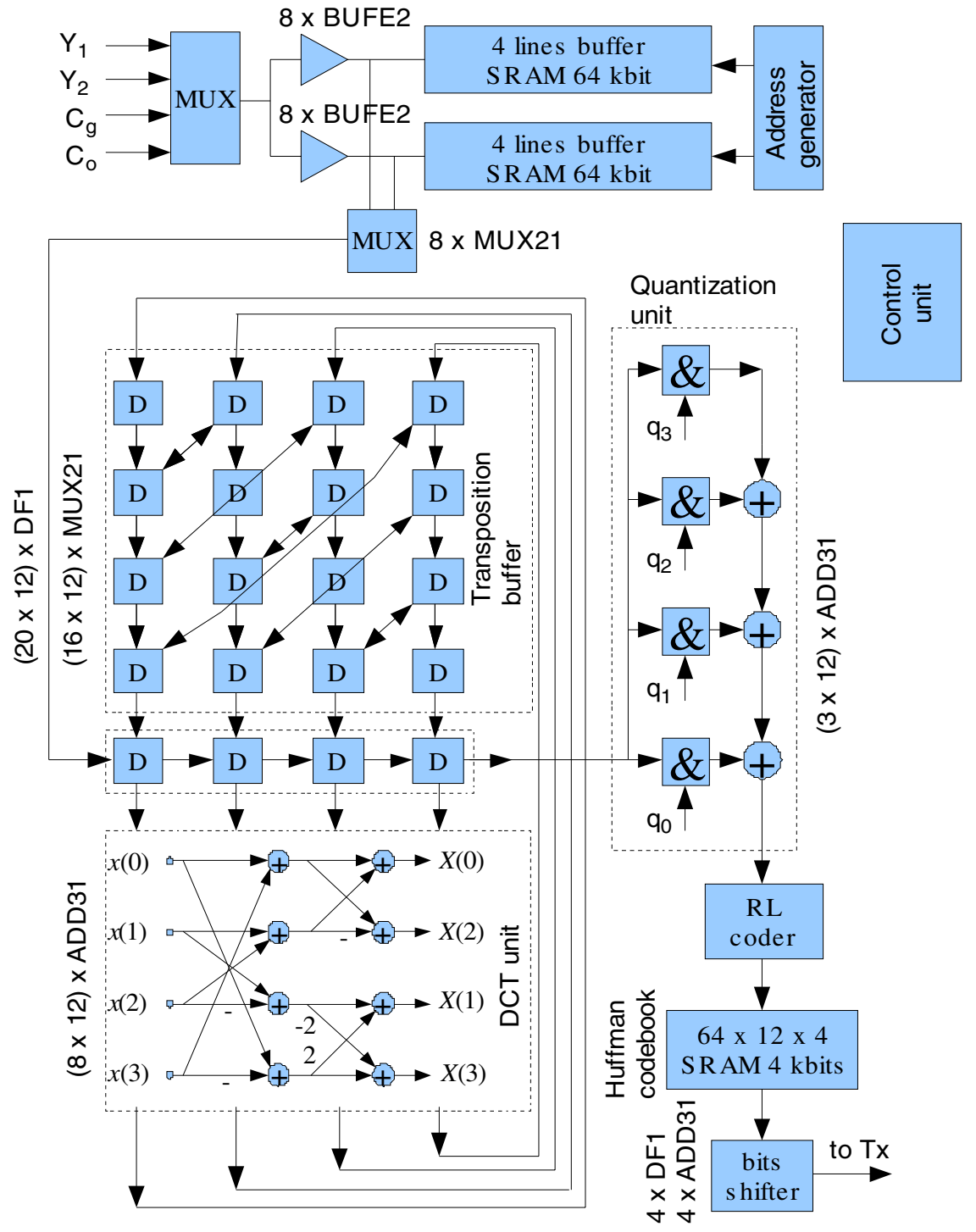

Fig. 6. Simplified VLSI architecture of the proposed image coder 
Table 1. Estimated power budget model [uA/MHz]

\begin{tabular}{|c|c|c|c|c|c|c|c|c|}
\hline \multirow[b]{2}{*}{ Module } & \multicolumn{4}{|c|}{ CMOS cell type } & \multicolumn{3}{|c|}{ SRAM } & \multirow{2}{*}{$\begin{array}{l}\text { Supply } \\
\text { current } \\
\mu \mathrm{A} / \mathrm{MHz}\end{array}$} \\
\hline & $\begin{array}{c}\text { DF1 } \\
0.345\end{array}$ & $\begin{array}{c}\text { ADD31 } \\
0.342\end{array}$ & $\begin{array}{c}\text { MUX21 } \\
0.148\end{array}$ & $\begin{array}{c}\text { BUFE2 } \\
0.254\end{array}$ & $\begin{array}{c}4 \text { kbits } \\
130\end{array}$ & $\begin{array}{c}8 \text { kbits } \\
160\end{array}$ & $\begin{array}{c}64 \text { kbits } \\
251\end{array}$ & \\
\hline Color trans. & 32 & 64 & - & - & - & 1 & - & 193 \\
\hline Line buffer & - & - & 8 & 16 & - & - & 2 & 503 \\
\hline DCT & 240 & 96 & 192 & - & - & - & - & 144 \\
\hline Quantizer & - & 36 & - & - & - & - & - & 12 \\
\hline Entropy coder & 4 & 4 & - & - & 1 & - & - & 132 \\
\hline TOTAL & & & & & & & & 984 \\
\hline
\end{tabular}

half band filter) and appropriate resampling. As an objective quality measure the peek signal to noise ratio (PSNR) has been used

$$
\mathrm{PSNR}=10 \log _{10} \frac{255^{2}}{m s e}, \quad m s e=\frac{1}{N \cdot M} \sum_{n=1}^{N} \sum_{m=1}^{M}\left(\hat{x}_{n, m}-x_{n, m}\right)^{2}
$$

where $\hat{x}_{n, m}$ denotes a value of the pixel having coordinates $(n, m)$ in reconstructed image (in Bayer CFA format).

Obtained compression ratio $(C R)$ with corresponding PSNR for different versions of algorithm based on DCT are presented in Fig. 9 and compared with the standard JPEG2000 coder. The following denotations are used in them: G1-G2-B-R independent coding of 4 images from CFA Bayer sensor having colors G1, G2, B, R the simplest approach; G12-B-R - coding without color transformation (2) but with structure transformation (Fig. 2); G12-B-R-di - coding without color transformation (2) but with structure transformation (Fig. 2) and additional deinterlacing/filtering (Fig. 3); Y12-U-V - coding with color transformation (2) and structure transformation (Fig. 2); Y12-U-V-di - coding with color transformation (2), structure transformation (Fig. 2) and additional deinterlacing /filtering (Fig. 3); Y12-U-VDCT8F - algorithm based on standard 8-point, floating-point DCT transform with color transformation (2) and structure transformation (Fig. 2).

We can conclude from Fig. 9 that:

1) due to aliasing and high-frequency image content created by the Bayer CFA sampling the $4 \times 4$ DCT algorithm slightly outperforms the JPEG2000 for lower compression ratios (e.g. 15) but it is worse than the JPEG2000 for higher ratios (e.g. 30 ); both algorithms have been defeated by the floating point $8 \times 8 \mathrm{DCT}$;

2) usage of color space conversion (2) is beneficial;

3) deinterlacing operation, described in Fig. 3, should be neglected since it makes the results worse.

In order to obtain results with higher statistical significance more extensive test on longer data set has been performed. 100 video frames have been chosen in random manner from gastroscopy/colonoscopy recordings and coded using Huffman tables with 64 entries (precomputed using different set of frames). From results presented in 


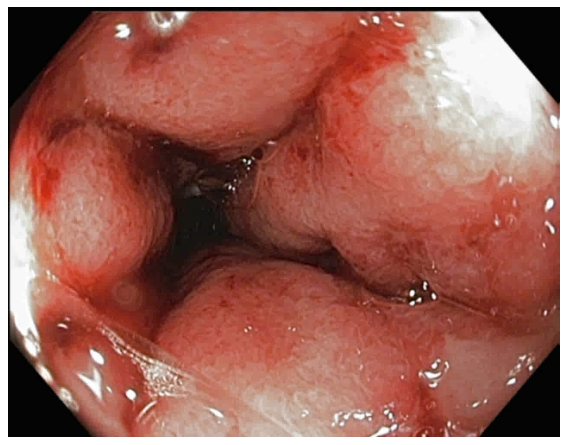

Fig. 7. Exemplary colonoscopy image

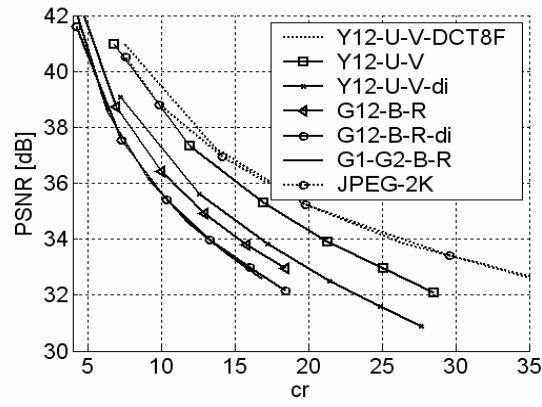

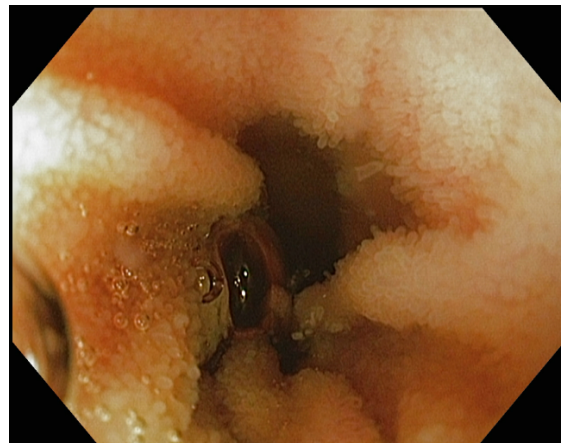

Fig. 8. Exemplary gastroscopy image

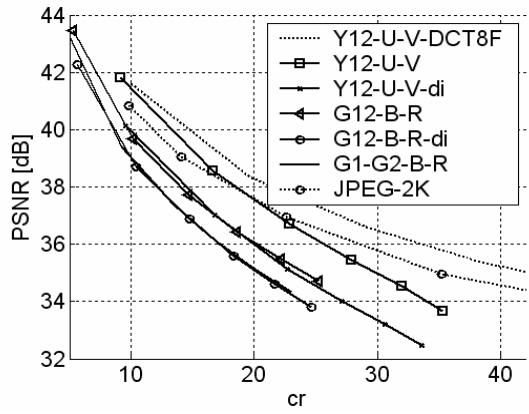

Fig. 9. Results for the DCT based image coder. (test images: left - colonoscopy, right gastroscopy).

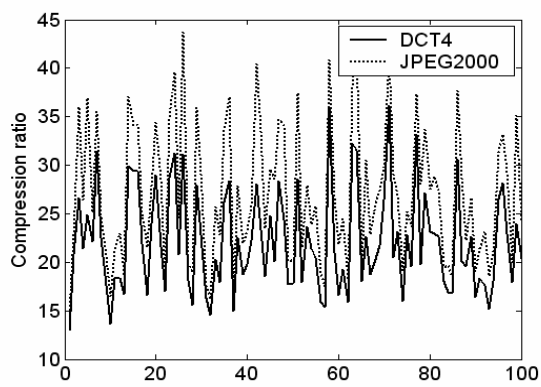

Fig. 10. Performance comparision

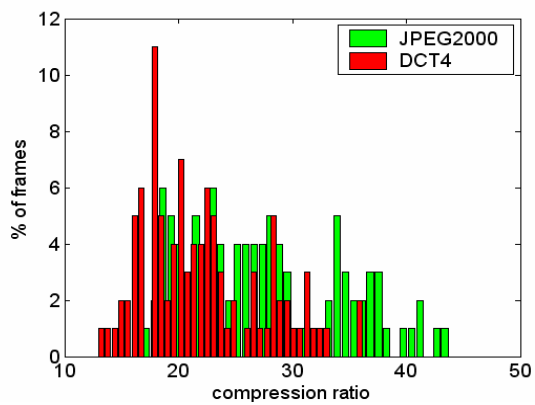

Fig. 11. Histograms of results from Fig. 10

Fig. 10, 11 we can conclude that for images with many diagnostic details the proposed compression scheme has efficiency similar to the JPEG2000 standard. The JPEG2000 algorithm is significantly better only for simple details-free images which do not have big diagnostic value. 


\section{Conclusions}

Low-complexity, low-power image compression algorithm suitable for wireless capsule endoscopy has been proposed and tested in the paper. It makes use of integer version of the discrete cosine transformation. Transform coefficients are encoded using optimized, low complexity Huffman coder.

Assuming that low implementation complexity and PSNR having at least $36 \mathrm{~dB}$ is required, the best PSNR-CR performance has been obtained for the DCT algorithm with the color space conversion (2) and structure conversion (presented in Fig. 2) but without image filtering/deinterlacing (shown in Fig. 3). For it we can get compression ratio $\mathrm{CR}=15$ for colonoscopy images and $\mathrm{CR}=25$ for gastroscopy ones.

Acknowledgments. The research activities presented in this paper were conducted under the European Commission R\&D Project No 3E061105 (VECTOR).

\section{References}

1. Iddan, G., Meron, G., Glukhovsky, A., Swain, P.: Wireless capsule endoscopy. Nature 6785, 417-418 (2000)

2. Mylonaki, M., Fritscher-Ravens, A., Swain, P.: Wireless capsule endoscopy: a comparison with push enteroscopy in patient with gastroscopy and colonoscopy negative gastrointestinal bleeding. Gut J., 1122-1126 (2003)

3. Turgis, D., Puers, R.: Image compression in video radio transmission for capsule endoscopy. Sensors and Actuators A, 129-136 (2005)

4. Xie, X., Li, G.L., Wang, Z.H.: A Near-Lossless Image Compression Algorithm Suitable for Hardware Design in Wireless Endoscopy System. EURASIP Journal on Advances in Signal Processing, Article ID 82160, 1-13 (2007)

5. Turcza, P., Duplaga, M.: Low-Power Image Compression for Wireless Capsule Endoscopy. In: IEEE Int. Workshop on Imaging Systems and Tech. - IST 2007, Krakow, Poland (2007)

6. Turcza, P., Zielinski, T., Duplaga, M.: Low complexity image coding algorithm for capsule endoscopy with Bayer color filter array. Signal Processing, Poland, Poznan, 27-32 (2007)

7. Bayer, B.E.: Color Imaging Array: U.S. Patent 3,971,065 (1976)

8. Koh, C.C., Mukherjee, J., Mitra, S.K.: New efficient methods of image compression in digital cameras with color filter array. IEEE Trans. on Consumer Electronics 49(4), 1448 1456 (2003)

9. ITU-T Rec. H.264 / ISO/IEC 11496-10: Advanced Video Coding, Final Committee Draft, Document JVTF100 (December 2002)

10. Malvar, H.S., Hallapuro, A., Karczewicz, M., Kerofsky, L.: Low-Complexity Transform and Quantization in H.264/AVC. IEEE Trans. on Circuits and Systems for Video Tech. 7 (2003)

11. Wallace, G.K.: The JPEG still picture compression standard. Communications of the ACM 34, 30-44 (1991) 